\title{
Chemical Analysis of Bioactive Compound Produced by Lactobacillusreuteri Isolated From Domestic Chickens in Iran
}

\author{
Baserisalehi majid*, Jamali fatemeh, Moradi parviz, Arzani parastoo, Shirali Masumen and Khalifehpour \\ Masood \\ Department of Microbiology, Islamic Azad University, Kazeroun, Iran
}

Received: December 07, 2017; Published: December 14, 2017

*Corresponding author: Majid Baserisalehi, Department of Microbiology, college of science, Kazeroun Branch, Islamic Azad University, Kazeroun, Iran

\begin{abstract}
The purpose was Chemical analysis of the bioactive compounds produced by Lactobacillus reuteri isolated from domestic chickens. In addition, the antimicrobial property of the bioactive compounds was assessed against pathogenic bacteria. A total twenty two samples were collected from digestive tracts of domestic chickens and subjected to microbiology analysis. The bioactive producing bacterium was identified and the bioactive compound was analyzed by SDS-PAGE and HPLC methods. Finally, antimicrobial property of the bioactive compound against the pathogenic bacteria was assessed. The results obtained indicated that the bioactive compound producing bacterium was Lactobacillus reuteri. The results obtained from SDS-PAGE indicated that the molecular weight of purified bioactive compound produced by the isolatewas3.5< $\mathrm{kDa}$. The bioactive compound showed antimicrobial effect against Escherichia coli, Salmonellatyphimurium, Staphylococcus aureus and Bacillus cereus. Salmonella typhimuriumi and cereus were more and less sensitive to the bioactive compounds. Overall, chemical analysis of the bioactive compounds illustrated that the bioactive compound produced by Lactobacillus reuteri was probably Lactocins.
\end{abstract}

Keywords: Lactobacillus spp; Bioactive Compound; Domestic Chickens

\section{Introduction}

Lactobacillus is a type of bacteria, which normally live in the intestinal tracts of some domestic animals and poultry. These bacteria are characterized by ability of producing different metabolites Aymerich et al. [1]. Some bioactive compound of Lactobacillus is proteinaceous in nature and mostly acts against closely related species Bali et al. [2]. Bioactive compounds could be produced by plants, animals and microorganisms. However, function of bioactive compounds is similar. But their structures and antimicrobial spectrum varied. Although, several reports conducted on production of bioactive compounds Ogunbanwo et al. [3] a few reports are available concerning to bioactive compounds produced by Lactobacillus. The present study was undertaken to isolate and identified bioactive producing Lactobacillus from the digestive tracts of domestic chickens, Furthermore, the antimicrobial property of bioactive compounds were assessed against pathogenic bacteria and their structures were chemically analyzed.

\section{Material and Methods}

\section{Sample Collection}

Twenty two samples were collected from the intestinal tracts of domestic chickens in south of Iran (Shiraz and Kazeroun) and subjected to microbiological analysis. The samples were serially diluted using sterile distillated water $\left(10^{-1}-10^{-4}\right)$ and $0.1 \mathrm{ml}$ of two last dilutions was streaked on MRS medium. The plates were kept in incubator at $37^{\circ} \mathrm{Cfor} 72 \mathrm{~h}$ Yodoamijoyo et al. [4].

\section{Phenotypic Identification of the Isolates and Screening of the Bioactive Producing Strains}

Phenotypic identification of isolates was carried out by Gram stain and catalase test and biochemical tests viz., hydrolysis of esculin, utilization of citrate, acid and gas formations from glucose , fermentation of D-xylose, galactose, D-fructose, sorbitol, lactose, melibiose, saccharose, Arabinose, Lactose, Mannose, Mannitol, Maltose, Raffinose, Sorbitol, Sucrose, Xylose, Melezitose, Trehalose, Rhamnose and Ribose and growth at different pH 4.5, 5.5 and 6.5 and temperatures25, 30, 35 and 40 Samelis et al. [5]. Screening of bioactive producing was carried out by cultivation of presumptive Lactobacillus strains into MRS broth. The bacterial suspensions were centrifuged at $4500 \mathrm{rpm}$ for $15 \mathrm{~min}$ and the supernatant of each isolates was assessed against the antagonistic bacteria such as Bacillus cereus (ATCC11778), Escherichia coli (ATCC29998), Salmonellatyphimurium (ATCC 14028), and Staphylococcus aureus (ATCC6538) by Well Agar Diffusion method. 


\section{Authentication of Lactobacillus Isolates by Gene Sequencing of 16SrRNA}

To perform the test universal primers of 27F (5'-AGAGTTTGATCMTGGCTCAG -3') and 1492R (5'-GGTTACCTTGTTACGACTT-3') was used Lane. Polymerase chain reactions were performed in $25 \mu \mathrm{l}$ reaction volumes containing $1 \mathrm{X}$ Taq Master $\mathrm{Mix}, 1.5 \mathrm{mM} \mathrm{MgCl}_{2}$, $0.25 \mathrm{mM}$ forward primer, $0.25 \mathrm{mM}$ reveres primer and $0.4 \mathrm{ng}$ of genomic DNA. Temperature cycling conditions for PCR were as follows: an initial heating of $95 \mathrm{oC}$ for $3 \mathrm{~min}$, followed by 30 cycles of denaturation at $950 \mathrm{C}$ for $30 \mathrm{~s}$, annealing at $55 \mathrm{oC}$ for $55 \mathrm{~s}$, extension at $72 \mathrm{oC}$ for $1 \mathrm{~min}$, and terminating with a $10 \mathrm{~min}$ final incubation of $72 \mathrm{oC}$. Gradient temperature PCR was utilized to improve primer-annealing temperatures to make certain a high degree of primer specificity during assays Shuhaimi [6]. The PCR products were examined by electrophoresis (Gel Electrophoresis Systems. Major Science, Taiwan) on a $1 \% \mathrm{w} / \mathrm{v}$ agarose gel, stained with ethidium bromide, visualized and photographed on a Gel Documentation. The PCR products were purified using a QI Aquick PCR purification kit (QIAGEN, Hilden, Germany) according to the manufacturer's applications. The purified PCR products were sent to BASE Laboratories, Malaysia. The 16SrRNA sequenced data were bioinformatic analyzed using http://www.ncbi.nlm.nih.gov/BLAST/

\section{Purification of the Bioactive Compounds}

Overnight cultures of the isolates were cultivated into MRS broth (10\% inoculums) $\left(10^{8} \mathrm{CFU} / \mathrm{ml}\right)$ and incubated for $72 \mathrm{hin}$ shaker incubator $(200 \mathrm{rpm})$ at $37^{\circ} \mathrm{C}$. The bacterial suspension of each strain was centrifuged at $12000 \mathrm{rpm}$ for $15 \mathrm{~min}$ and the supernatant was used as crude bioactive compounds. The supernatant was separated and saturated with $70 \%$ ammonium sulfate and stored at $4^{\circ} \mathrm{C}$ to precipitate the proteins. The pellet was collected after centrifugation at $12000 \mathrm{rpm}$ at $4^{\circ} \mathrm{C}$ for 25 minutes and dissolved in a phosphate buffer $(0.1 \mathrm{M}, \mathrm{pH} 7.0)$ and dialyzed against the same buffer at $4{ }^{\circ} \mathrm{C}$ overnight Ogunbanwo et al. [3]. The dialyzed protein was applied to a Sephadex G-100 column (1.6 × $36 \mathrm{~cm})$, pre-equilibrated with phosphate buffer $(\mathrm{pH} 7.0)$ and the fractions showing high bioactive compound activity were collected and subjected to chemical analysis.

\section{Chemical Analysis of the Bioactive Compounds by SDS- PAGE and HPLC}

The molecular weight of the purified bioactive compound was determined by $15 \%$ sodium dodecyl sulfate polyacrylamide gel electrophoresis (SDS-PAGE) according to the method described by Laemmli in 1970. The electrophoresis gel was stained with Coomassie Brilliant Blue R-250 and washed by the mixture of acetic acid-methyl alcohol-water (5:5:1 v/v). The low range molecular weight marker was used as a standard marker (Genei, India). To perform HPLC, the bioactive compounds were centrifuged at $12000 \mathrm{rpm}$ for $15 \mathrm{~min}$. Then supernatant was filtered through a $0.22 \mu \mathrm{m}$ filter and injected into HPLC system. The analysis of the bioactive compounds was carried out by HPLC system (Shimadzu, Japan) with a UV-VIS multi wavelength detector. A HPLC column was $\mathrm{C}_{18}, 250 \times 4.6 \mathrm{~mm}, 5 \mu \mathrm{m}$ particle size, Sun Fire and the mobile phase of Chromatographic conditions was phosphate buffer 0.01
mol/L-3.0\% methanol, $\mathrm{pH} \mathrm{3}$, detection at $210 \mathrm{~nm}$ of Ultraviolet (UV) absorbance, $0.7 \mathrm{~mL} / \mathrm{min}$ flow rate and $10 \mu \mathrm{l}$ injection volume. The column temperature was kept ambient. The mobile phase was prepared and filtered through a $0.22 \mu \mathrm{m}$ filter membrane.

\section{Results}

\section{Identification of the Bioactive Compounds Producing Lactobacillus}

A total, 6 strains of Lactobacillus were isolated based on phenotypic identification. Of all, one strains of Lactobacillus could produce the bioactive compound. The bioactive compound produced by this strain showed antimicrobial effect against Escherichia coli, Salmonellatyphimurium, Staphylococcus aureus and Bacillus cereus.

\section{Molecular Identification of the Bioactive Compound Producing Lactobacillus}

The bioactive producing strain of Lactobacillus was subjected to 16SrRNA Gene sequencing. The results obtained indicated that bioactive producing Lactobacillus was Lactobacillus reuteri.

\section{The chemical analysis of the purified bioactive compound produced by Lactobacillus reuteri}

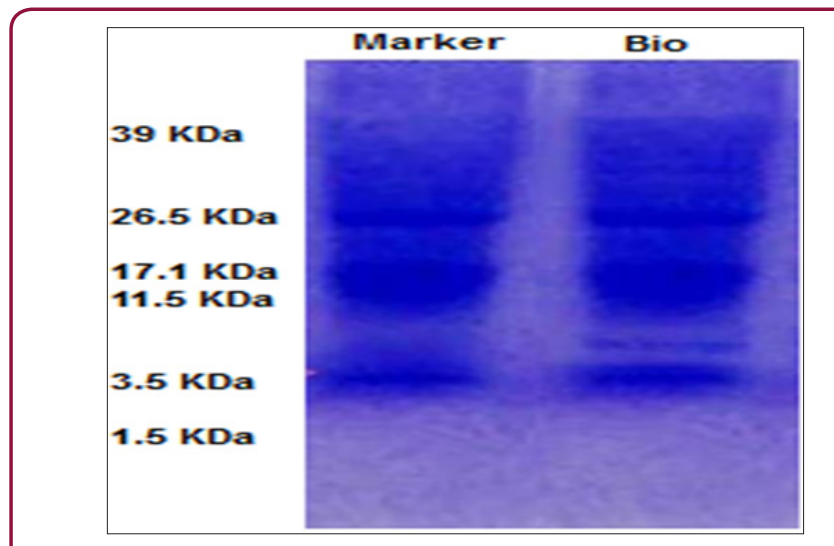

Figure 1: SDS PAGE of the bioactive compound produced by $L$ reuteri.

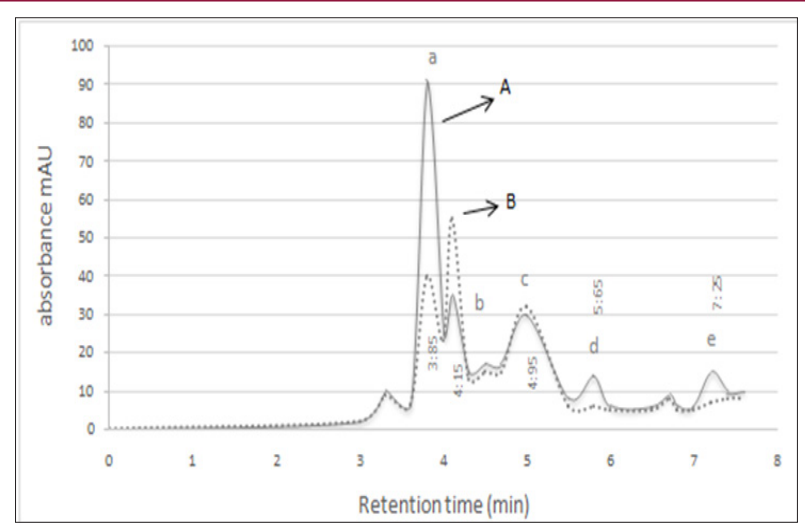

Figure 2: HPLC chromatograms.

A) Standard solutions of organic acids,

B) Detection of organic acids produced by L . reuteri

a. Malic acid,

b. Lactic acid,

c. Acetic acid. 
The results obtained from SDS-PAGE indicated that the molecular weight of purified bioactive compound produced by $L$ reuteri were $4.5 \mathrm{kDa}$ similar to Lactocin (Figure 1). HPLC was done on the bioactive compound obtained verified presence of some organic acids include in the bioactive compound. Chromatograms of HPLC were shown in (Figure 2). As seen in these figure three organic acids were recognized based on their polarity and sizes. These organic acids were malic acid, lactic acid, and acetic acid. Retention times of standard samples such as malic acid, lactic acid and acetic acid were 3.85, 4.15, 4.95 and 5.65minutesrespectively.

\section{Discussion}

Lactobacillus spp. are recognized as major bioactive producing bacteria that especially living in intestinal tracts of chickens. In addition, Lactobacillus strains have several properties viz., resistance to gastric and intestinal juices and ability to adhere to intestinal surfaces Patterson and Burkholder [7]. It must be noted that property of Lactobacillus spp. might be depended on geographical areas. For instant, response of Lactobacillus to osmotic pressure is not similar Ben et al. [8]. Therefore the present study was purposed to isolate Lactobacillus from domestic chicken and determine the chemical properties of their bioactive compounds. A total one bioactive compound producing strain of Lactobacillus was isolated and recognized as lactobacillus reuteri K16. The bioactive compounds produced by this bacterium showed antimicrobial effect against Escherichia coli, Salmonella typhimurium, Staphylococcus aureus and Bacillus cereus. Chemical analysis of the bioactive compound produced by lactobacillus reuteri isolate indicated that SDS-PAGE and HPLC methods detected the compounds most likely Lactocin (molecular weight of $4.5 \mathrm{kDa}$ ) and organic acids viz., malic, lactic and acetic acids. Parallel with our finding Lasta et al. [9,10] isolated Lactocin producing lactobacillus. However their isolate was Lactococcus lactis. Overall our investigate opined that domestic strains of lactobacillus could produce bioactive compound similar to
Lactocin. Hence, Lactobacillus spp. isolated from each geographical area must be evaluated for probiotic property. It is because probably domestic probiotic might be have more advantage compared to commercial probiotics.

\section{References}

1. Aymerich T, Artigas MG, Monfort JM, Hugas M (2000) Effect of sausage ingredients and additives on Cuban. Journal of Agricultural Science 46: 8.

2. Bali V, Panesar PS, Bera MB (2011) Isolation, screening and evaluation of antimicrobial activity of potential bacteriocin producing lactic acid bacteria isolate. Microbiol J 1: 113-119.

3. Ogunbanwo ST, Sanni AI, Onilude AA (2003) Characterization of Bacteriocin Produced by Lactobacillus plantarum F1 and Lactobacillus brevis OG1. African Journal of Biotechnology 2(8): 219- 227.

4. Yodoamijoyo RM, Tirza Z, Harastuti Tomomatsu A Matsuyama A, Hosono A Antimicrobiol activities of Lactic acid bacteria strains. J dairy food Sci 32: 7-14.

5. Samelis J, Maurogenakis F, Metaxopoulos J (1994) Characterization of lactic acid bacteria isolated from naturally fermented Greek dry salami. Int J Food Microbiol 23(2): 179-196.

6. Shuhaimi M (2003) Species classification and molecular studies of bile salt hydrolase gene in Bifidobacterium spp. Doctor of philosophy. University Putra Malaysia 1-174.

7. Patterson JA, Burkholder KM (2003) Application of prebiotics and probiotics in poultry production. Poult Sci 82(4): 627.

8. Ben CP, Beula A, Nirmala Devi S (2012) Production and Partial Purification of Lactocin produced from Lactobacillus lactis and its Bactericidal activity against Food spoiling bacteria. ISCA Journal of Biological Sciences 1(3): 49-53.

9. Lasta S, Ouzar H (2012) Lactocin LC14, a New Bacteriocin Produced by Lactococcus lactis BMG6.14: Isolation. Purification and Partial Characterization P 3(2): 133-13.

10. Laemmli UK (1970) Cleavage of structural proteins during the assembly of the head of bacteriophage T. 4 Natures 277 (5259): 680-685.

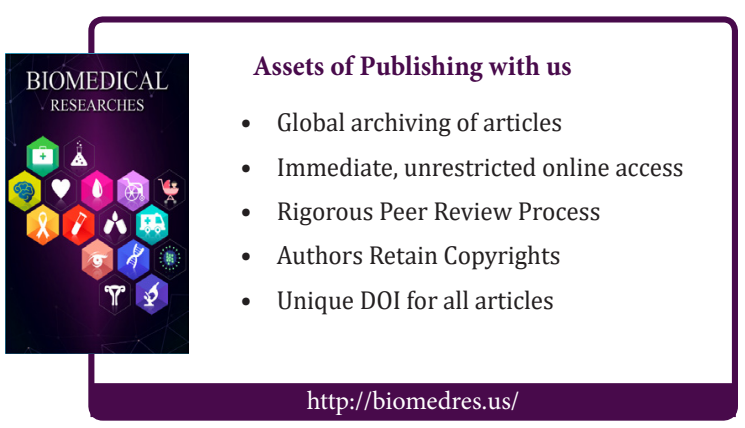

